\title{
Raising students motivation for math learning using computer animation approach
}

\author{
Manuel J. Ibarra' ${ }^{1}$ Yonatan Mamani ${ }^{1}$, Pablo E. Ataucusi ${ }^{2}$, Carmen Palomino ${ }^{3}$, Vladimiro Ibañez ${ }^{4}$ \\ Universidad Nacional Micaela Bastidas de Apurímac - Perú1, Colegio de Alto Rendimiento \\ Apurímac - Perú ${ }^{2}$, Universidad Tecnológica de los Andes-Perú ${ }^{3}$, Universidad Nacional del \\ Altiplano-Perú ${ }^{4}$ \\ manuelibarra@gmail.com, ymamanic@gmail.com, elicoena@ hotmail.com, \\ magcarmenpalomino@ hotmail.com, viqibanezquispe@gmail.com
}

\begin{abstract}
This article aims to improve the math learning process of the students in rural schools. For this purpose, a "Mathfraction" computer animation application was developed to use on the web and mobile technologies. The tool was designed using a visual approach to reinforce student's learning process acquired in classes. The tool was tested and validated by three usability user experts, thirty four students and four teachers of two rural schools in Apurímac-Perú. Three types of tests were performed, the first one was a server's performance test, the second one was using a user usability questionnaire and the last one was by user expert opinion questionnaire. The results of this research show that teachers and students agree that Mathfraction allows them to teach and learn math in a fun way and different from the other traditional methodologies that they knew.
\end{abstract}

Index Terms - Computer Animation, Computer Visual Approach, Fractions, Human Computer Interaction, Math Learning

\section{INTRODUCCIÓN}

$\mathrm{E}^{\mathrm{n}}$ n Latinoamérica, Perú ocupa los últimos lugares en evaluación en matemáticas y comunicación, así lo muestran las pruebas internacionales realizadas por PISA [1] y LLECE [2]. En el Perú el Ministerio de Educación mediante la Unidad de Medición de la Calidad Educativa ha llevado a cabo la Evaluación Censal de Estudiantes (ECE) [3], los resultados obtenidos por la ECE muestran que existe una relación entre el nivel socioeconómico y los puntajes obtenidos por los estudiantes; por un lado, Lima Metropolitana y Callao con mayor puntaje; por otro lado, Ancash, Madre de Dios, Loreto y Apurímac con menor puntaje. Asimismo, los resultados de la ECE también muestran que existen diferencias muy marcadas entre los logros alcanzados entre las Instituciones Educativas urbanas y rurales.

Las Tecnologías de Información y Comunicación (TIC) son el conjunto convergente de tecnologías que permiten la adquisición, producción, almacenamiento, tratamiento, comunicación, registro, acceso y presentación de datos y contenido [4]. El avance de las TIC en el campo educativo, en concreto en la didáctica de las matemáticas, está ocasionando importantes modificaciones en la labor del docente. La forma tradicional de la enseñanza de la matemática con pizarra, tiza y papel está pasando a ser un espacio ocupado también por equipos informáticos y conexión de banda ancha. Lo cierto es que las TIC han logrado insertarse en la sociedad actual y transformarla de acuerdo a sus necesidades y alcances [5], [6]. Sin embargo, ipueden producir las TIC, por sí mismas, un cambio sustancial en la mejora de la calidad de la enseñanza y consecuentemente del aprendizaje de los alumnos?, es evidente que no, se requiere que estén acompañadas de un cambio sustancial en la dirección del proceso de enseñanzaaprendizaje y de un perfeccionamiento de la didáctica, esto ha sido ampliamente discutido por investigadores de la especialidad [7], [8] y [9].

Los educadores coinciden manifestar que el objetivo más importante de la enseñanza de las matemáticas debe ser que los estudiantes desarrollen la capacidad de "comprender", aunque puede ser difícil conceptualizar este entendimiento en su totalidad [10] y [11]. El reto de la comunicación es un aspecto aún más preocupante por los problemas de vocabulario y claridad, porque existe la tendencia de pensar que las descripciones verbales constituyen conocimiento [12] y que la abstracción matemática es esencialmente verbal.

En las matemáticas, uno de los conceptos en el cual los alumnos presentan diversas dificultades en su comprensión, es en "las fracciones". Los problemas más frecuentes que se presentan al enseñar las fracciones son: se centran en el conteo de partes priorizando el número de partes y no la relación entre la parte y el todo; no se trabaja en la independencia de una forma simple; no se trabaja con fracciones mayores que la unidad; no se hace hincapié en la relación número de partes y tamaño de las mismas; no se representan distintas fracciones en una misma unidad [13], [14], [15], [16], [17], [18] y [19]. Uno de los factores que posiblemente incide en este proceso es la didáctica tradicional empleada en la enseñanza, la cual sería uno de los más determinantes del ulterior aprendizaje del niño [20], [21], [22], [23]. El desafío es hacer que la enseñanza de las matemáticas sea más intuitiva, dinámica, participativa, motivadora y divertida.

El uso de la computadora está abriendo espacios para que el estudiante viva nuevas experiencias dentro del 
mundo de las matemáticas (difícilmente de lograr en medios tradicionales como pizarra, plumón, papel, lápiz), y le permita manipular directamente los objetos matemáticos en un ambiente de exploración [24], [25], [26], [27], [28]. Durante los últimos años los investigadores del área computación y didáctica de la matemática han identificado la necesidad de encontrar nuevas estrategias para la enseñanza de las matemáticas, enfocándose básicamente en hacerlo en forma lúdica, utilizando animaciones por computadora, con representaciones visuales y de acuerdo al contexto de los alumnos [29], [30], [31] y [32].

En matemáticas muchos conceptos y procesos podrían ligarse a interpretaciones visuales, lo que ha generado diversas investigaciones en relación con el potencial didáctico de la visualización, la forma como ésta podría favorecer al aprendizaje y bajo qué condiciones utilizarla [33], [34]. La visualización implica que el profesor induzca a los estudiantes a formarse una imagen del problema, a describirlo con sus propias palabras, creando una imagen o modelo tridimensional e incluso darle movimiento a la situación del problema [35]. La representación visual es una de las herramientas efectivas para crear conceptos, y con el avance de la tecnología se ha incluido animaciones por computadora para la enseñanza de las matemáticas [36].

Este artículo presenta una estrategia (complementaria a la forma tradicional) para enseñar las "Fracciones" en el área de matemática, esto está orientado para el nivel primario de los colegios en Apurímac-Perú, se caracteriza por tener un enfoque visual mediante animación por computadora, de tal manera que sea motivadora, lúdica y divertida para los alumnos.

La sección II del presente artículo presenta y discute los trabajos relacionados referidos al tema de enseñanza de las matemáticas y en especial de las fracciones; la sección III explica el diseño e implementación de la estrategia propuesta; la sección IV explica la metodología de evaluación utilizada para validar la estrategia propuesta; finalmente, la sección $\mathrm{V}$ describe las conclusiones y trabajo futuro de esta investigación.

\section{Trabajos Relacionados}

Chuang [37] realizó un estudio denominado "Teaching in a Multimedia Computer Environment: A Study of the Effects of Learning Style, Gender, and Math Achievement", en dicho estudio se analizó los efectos que producen los estilos de aprendizaje. Los estilos de aprendizaje se dividen de la siguiente forma: en el primer caso es un aprendizaje basado sólo en texto; en el segundo caso es un aprendizaje mediante una narración oral; en el tercer caso es mediante una animación por computadora. Para realizar el experimento se crearon 4 cursos, el primero fue denominado animación+texto, el segundo animación+voz, el tercero animación+texto+voz, y una cuarta denominada libre para que el estudiante seleccione. La investigación mostró que los alumnos que utilizaron el estilo de aprendizaje mediante la animación+texto+voz obtuvieron mejores resultados y fue la interfaz preferida por los estudiantes.

Por otra parte Chang [29] realizó una investigación en la cual describe las consideraciones que se deben tener en cuenta para la enseñanza con enfoque visual utilizando computadoras. El estudio menciona que con el desarrollo tecnológico, la masificación de las computadoras y los avances de las ciencias de la computación, el aprendizaje mediante la visualización juega un rol importante en el proceso de enseñanza. El artículo se centra en las dificultades que se presentan al poner en práctica la enseñanza por medios visuales, y concluye que los aspectos importantes a considerar son: el diseño del software, la integración cultural, el aspecto cognitivo, el principio del canal dual en el procesamiento de la información, la efectividad de las aplicaciones desarrolladas.

Similarmente Félix y otros [38], realizaron un estudio denominado "Objeto de Aprendizagem para o ensino da $\mathrm{Ma}$ temática", para realizar el trabajo de investigación crearon un juego educativo para niños de enseñanza básica en Brasil, la aplicación fue desarrollada para aprender los números naturales y funciona en dispositivos móviles con sistema operativo Android. Los resultados de esta investigación muestran que se creó un software educativo para enseñar matemática a los niños, este software estimula y enseña en forma lúdica el proceso de enseñanza aprendizaje. El software fue probado y validado por personas de edades superiores a las del público objetivo, esto para garantizar que el juego cumpla con el objetivo principal de motivar que los niños lo usen.

Desde el enfoque visual y multimodal, Valdez y otros [39], realizaron la investigación lleva por título "Math Snacks: Using Animations and Games to Fill the Gaps in Mathematics". En este trabajo se realizó un software llamado Math Snack, la animación funciona tanto en la web como en dispositivos móviles con sistema operativo Android. Los temas que se desarrollaron utilizando la animación fueron: radio, proporción, factor, escala y números. Este estudio piloto realizó la comparación de los resultados de pre y post test, consideró a 460 alumnos del sexto y sétimo grado de nueve diferentes salones. En cinco salones se utilizó Math Snack y su respectiva guía del profesor para trabajar con animaciones y juegos interactivos, y en los cuatro restantes salones no se utilizó el software. Los resultados finales muestran que los alumnos del sétimo grado que utilizaron el software y las guías obtuvieron mejores resultados respecto a los que no lo utilizaron.

El uso de las TIC en el aula hoy en día se ha convertido en una herramienta muy importante, Aguiar [40] realizó una investigación denominada "As novas tecnologias e o ensino-aprendizagem", cuyo objetivo fue analizar las innovaciones pedagógicas necesarias en el aula utilizando las Nuevas Tecnologías de Información y Comunicación, en la cual es necesario re-pensar las prácticas pedagógicas que el maestro debe aplicar en las aulas, especialmente para la enseñanza de las matemáticas.

El enfoque de la enseñanza utilizando animaciones han sido analizadas por varios investigadores, así por ejemplo Rahmat [41] realizó una investigación titulada "The impact of computer animation learning toward students academic performance", esta investigación analiza el impacto que tiene el uso de la computación animada en el proceso de aprendizaje y el rendimiento académico de los 
alumnos, el estudio se realizó en Malasia y los resultados muestran que el uso de la computación animada parece ser un factor poderoso al diseñar material pedagógico para los alumnos.

Asimismo, Aloraini [42] realizó una investigación denominada "The impact of using multimedia on students' academic achievement in the College of Education at King Saud University", el propósito de esta investigación fue encontrar el uso de la multimedia en alumnos que estudian cursos de informática en la facultad de Educación de la universidad de King Saud, los resultados muestran que el uso de multimedia en las sesiones didácticas dan mejores resultados que los que no lo usan.

Moyer-Packenham and Suh [43] el trabajo "Learning Mathematics with Technology: The Influence of Virtual Manipulatives on Different Achievement Groups" esta investigación destaca el uso de las manipulaciones virtuales (usando applets) en el aprendizaje de las matemáticas.

De acuerdo a la revisión bibliográfica realizada, se puede apreciar que efectivamente hay algunos avances en el uso de la computadora para la enseñanza de la matemática. Este artículo presenta una estrategia de enseñanza de las matemáticas, en especial del tema de fracciones, que complementa el modelo de enseñanza tradicional y utilizando un enfoque visual denominado animación por computadora.

\section{Diseño e IMPLementación de LA ESTRATEGia PROPUESTA}

\subsection{Arquitectura del sistema}

Los usuarios (Alumnos y Docentes) pueden utilizar la aplicación de tres formas: a) desde el laboratorio de cómputo de la Institución Educativa, en el cual se tiene computadoras conectadas con una topología estrella dentro de una red LAN (usuario color rojo); b) desde el campus de la Institución Educativa mediante una conexión WiFi (usuario color verde); c) desde su casa o una cabina de internet pública (usuario color azul), ver Fig. 1.

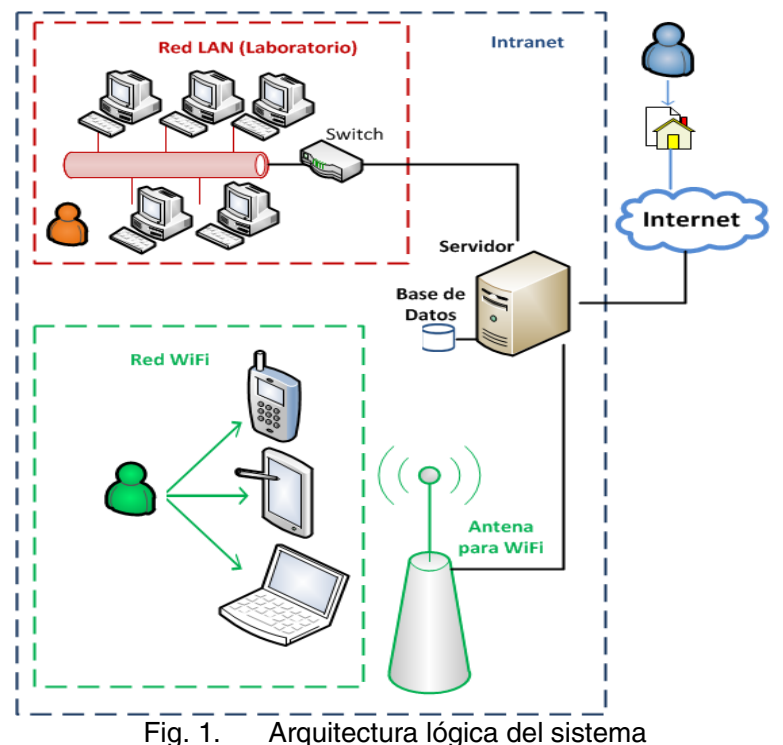

Los usuarios que utilizan una computadora personal con conexión al servidor, utilizan un navegador web e invocan a una página web mediante el protocolo HTTP, esta página web reside en el servidor web, que a su vez peticiona a los recursos necesarios para dar respuesta a la información solicitada por el cliente. Los usuarios que utilizan un dispositivo móvil sin conexión a la red interna, necesitan descargar un archivo del servidor e instalarlo en el celular o tablet, el instalador está disponible para dispositivos móviles con Sistema Operativo Android.

\subsection{Herramientas utilizadas en el desarrollo del software}

El software desarrollado está elaborado utilizando las siguientes herramientas:

-Action Script 3.0

Es un lenguaje de programación de la plataforma Adobe Flash, sirve para desarrollar aplicaciones web dinámicas y soporta programación orientada a objetos. Flash está compuesto por objetos con su respetiva ruta dentro del swf. Cada uno de estos objetos en ActionScript pertenece a una clase MovieClip, Botones, Vectores, Arrays, etc., que contiene propiedades, métodos o funciones.

-HTML.

Siglas de HyperText Markup Language ("lenguaje de marcas de hipertexto"), hace referencia al lenguaje de marcado para la elaboración de páginas web. Es un estándar que sirve de referencia para la elaboración de páginas web en sus diferentes versiones, define una estructura básica y un código (denominado código HTML) para la definición de contenido de una página web, como texto, imágenes, videos, entre otros.

-Apache web server

Es un servidor de páginas web, basado en el protocolo HTTP de código abierto, para plataformas Unix (BSD, GNU/Linux, etc.), Microsoft Windows, Macintosh y otras, que implementa el protocolo HTTP/1.1.

\subsection{Interfaz del software desarrollado}

La aplicación desarrollada inicia con un menú gráfico, en el cual se puede visualizar 4 opciones, ver Figura 2. Cada una de las opciones del menú está diseñada para enseñar el tema de fracciones de una manera distinta, visual y divertida. La primera está orientada para que el alumno pueda determinar gráficamente el numerador y denominador de una fracción; la segunda, para ubicar gráficamente una fracción en la recta numérica; la tercera es para realizar operaciones básicas con fracciones; y cuarta para relacionar las fracciones con porcentajes. 


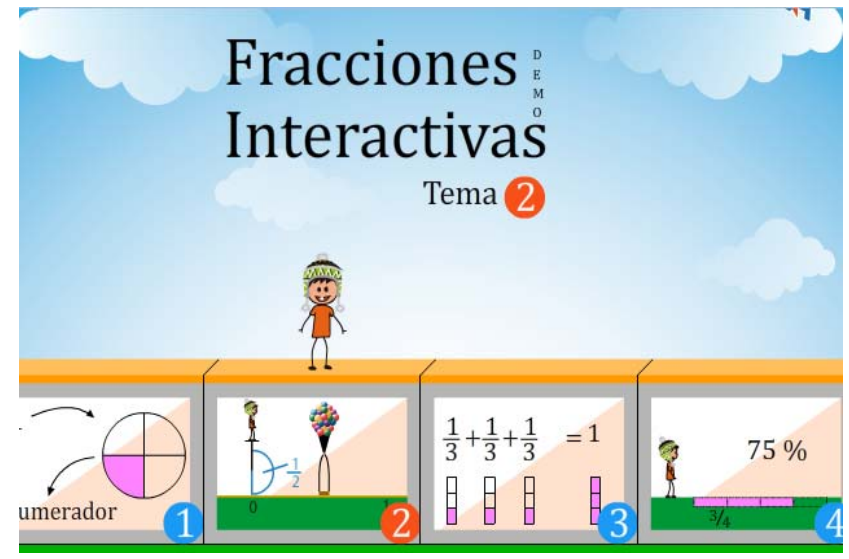

Fig. 2. Menú principal

Una vez escogida una de las opciones del menú, aparece un submenú con las opciones para elegir, ver Figura 3.

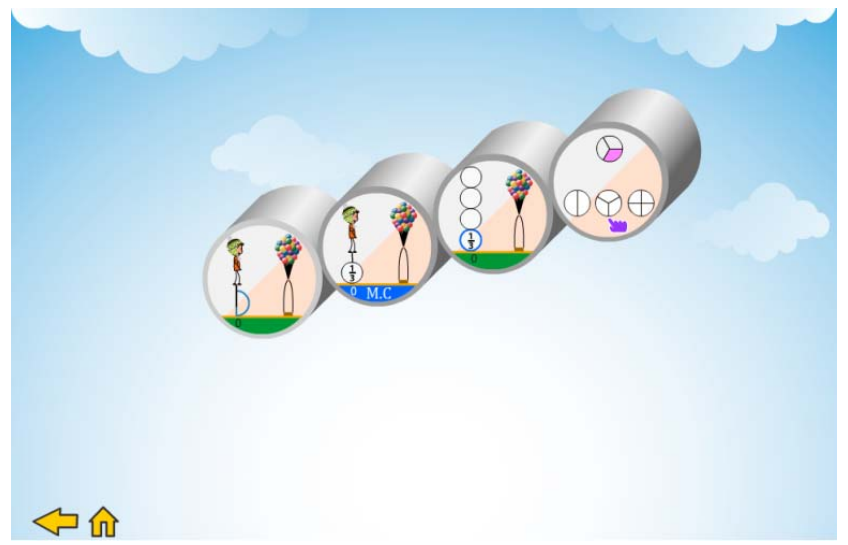

Fig. 3. Opciones del sub-menu

Después de seleccionar el sub-menú, el usuario puede escoger el nivel de dificultad que desea, ver Figura 4.

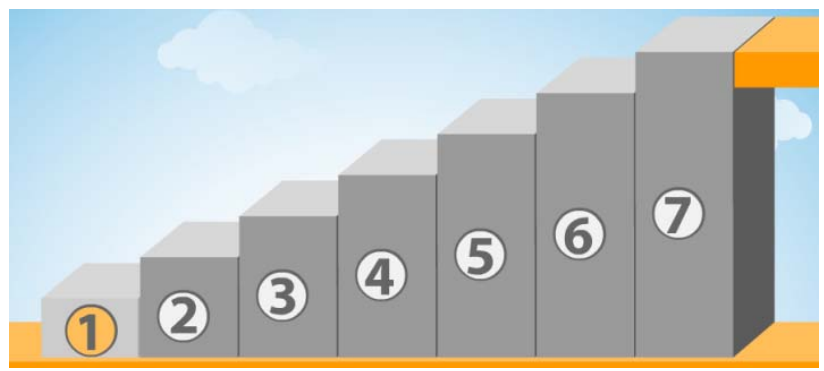

Fig. 4. Niveles de dificultad

Luego el usuario debe visualizar las circunferencias que le muestra en el lado izquierdo y mover los globos a la ubicación correcta, para el ejemplo que muestra la figura 5, la fracción mostrada en el lado izquierdo es "uno y medio", entonces el usuario debe mover los globos hasta la posición uno y medio. En caso que sea correcto, entonces el programa emitirá un sonido indicando que la posición marcada es correcta; en caso que no haya colocado en la posición correcta, entonces el programa le dará otra opción para completar el nivel.

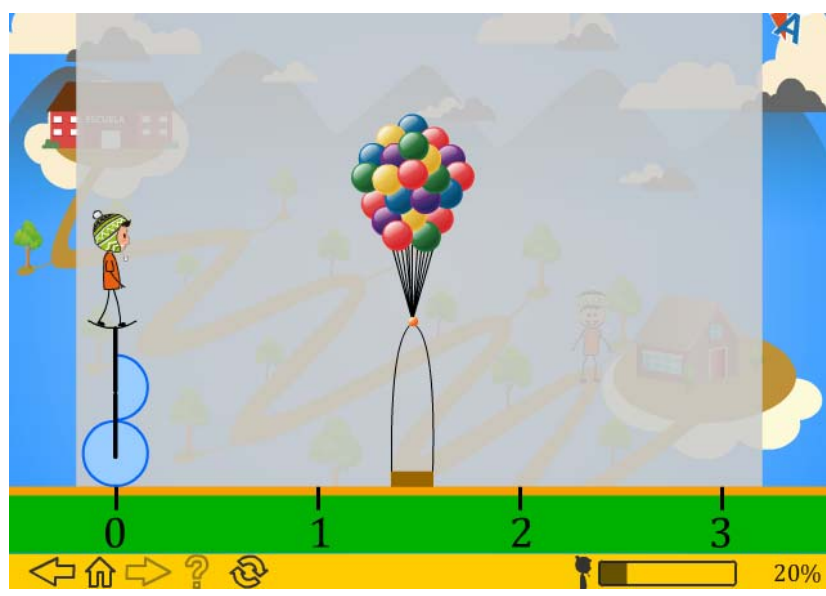

Fig. $5 . \quad$ Juego interactivo

En la parte inferior izquierda de la pantalla (ver Figura 5) muestran los botones para regresar al nivel anterior, para ir al inicio, para avanzar al siguiente nivel, para mostrar la ayuda y para actualizar el juego. En la parte inferior derecha de la pantalla muestra el porcentaje de avance del nivel en el que se encuentra (por ejemplo 20\%) y cuando dicho porcentaje llegue a 100, entonces pasará al siguiente nivel.

Cuando el usuario pasa al siguiente nivel, entonces un marcador gráfico le indicará la distancia que le falta para concluir el nivel, y es la distancia desde la posición del hombre hasta la posición de la Escuela y cuando el "hombrecito" llegue a la Escuela, entonces habrá terminado el juego y pasará al siguiente nivel, ver Figura 6.

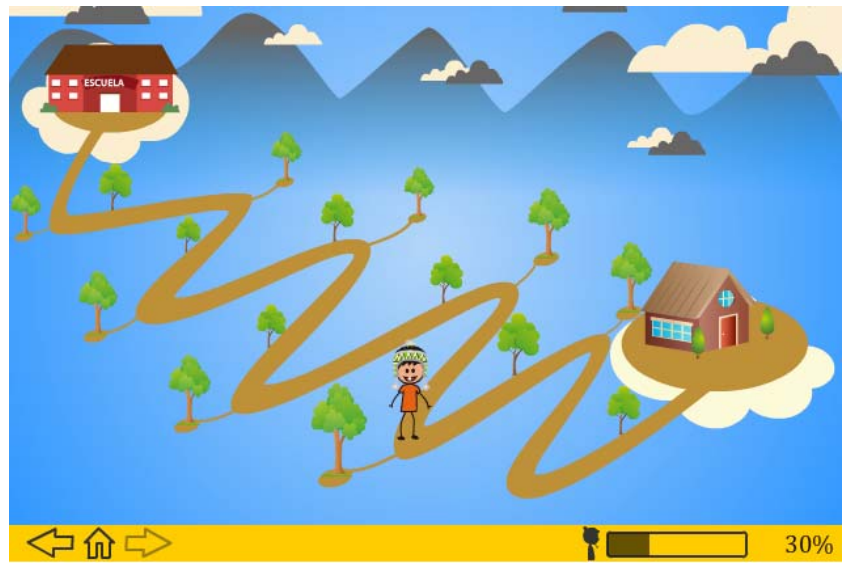

Fig. 6. Porcentaje de avance del nivel seleccionado

\subsection{Uso de un archivo de configuración "XML"}

Para mejorar la calidad del software y mejorar el mantenimiento de la aplicación propuesta, se ha visto por conveniente utilizar un archivo de configuración en XML, para que el programa pueda crear fácilmente las escenas de acuerdo al nivel de dificultad. La Figura 7 muestra el archivo "config.xml" con los parámetros iniciales. 


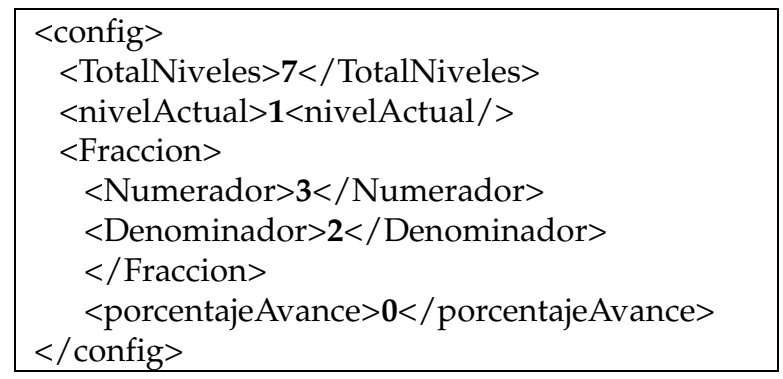

Fig. 7. Configuración inicial del juego interactivo

\subsection{Despliegue de la aplicación para diferentes plataformas}

Adobe AIR Runtime, es una tecnología enfocada a la creación de aplicaciones de escritorio a partir de tecnologías de desarrollo de páginas web como puede ser HTML, Ajax o Flash. Una de las ventajas adicionales es que las aplicaciones generadas son multiplataforma, es decir, se puede compilar la aplicación para Android, Mac iOS o Windows Mobile. Esta aplicación desarrollada se ha empaquetado sólo para dispositivos móviles con Android.

\section{PROCESO DE EVALUACIÓN DE LA ESTRATEGIA PROPUESTA}

Para dar validez a este trabajo de investigación se realizaron tres tipos de evaluaciones. La primera fue realizar pruebas de rendimiento a la aplicación desarrollada, la segunda fue aplicar un cuestionario de usabilidad a los usuarios finales y la tercera mediante opinión de usuario experto.

\subsection{Pruebas de rendimiento}

Para ver el rendimiento del servidor web, se realizaron "pruebas de carga" para medir el rendimiento de la memoria, el disco y la saturación de la aplicación (cuando esté en funcionamiento real).

Las pruebas de carga se realizaron con la herramienta Apache Bench, y para hacer la prueba de rendimiento se utilizó el comando:

$a b-n 1000$-c 200 http://192.168.1.35/mathfraction/index.html donde $a b$ es el comando que ejecuta Apache Bench; $-n$ 1000 es el número de peticiones que se requiere ejecutar; c 200 es el número de usuarios concurrentes que acceden a la página y finalmente se coloca la URL de la página en prueba.

Asimismo, para realizar las pruebas de carga, se utilizó una computadora personal convencional que hace las veces de servidor web, que tiene las siguientes características: Procesador Core i3, Unidad Central de Proceso de 3.40 GHz, 4Gb de memoria RAM, 500GB de disco duro, y otros dispositivos adicionales como monitor, teclado, mouse, etc. En el servidor antes mencionado, se instaló el sistema operativo Windows 7, un servidor web HTTP Apache de código abierto. Los resultados de las pruebas realizadas en el servidor web antes mencionado, se muestran a continuación en la tabla 1:
TABLA 1

Resultados de las pruebas de carga

\begin{tabular}{|l|r|r|r|r|r|}
\hline & $\begin{array}{l}\text { Nro. } \\
\text { Petic. }\end{array}$ & $\begin{array}{l}\text { Petic. } \\
\text { concurr. }\end{array}$ & $\begin{array}{l}\text { min. } \\
\text { (ms) }\end{array}$ & $\begin{array}{l}\text { prom. } \\
\text { (ms) }\end{array}$ & $\begin{array}{l}\text { max. } \\
\text { (ms) }\end{array}$ \\
\hline connect & 1000 & 10 & 15 & 227 & 9226 \\
\hline processing & 1000 & 10 & 188 & 2049 & 13115 \\
\hline waiting & 1000 & 10 & 187 & 1618 & 10044 \\
\hline connect & 1000 & 50 & 0 & 105 & 9110 \\
\hline processing & 1000 & 50 & 31 & 5158 & 28149 \\
\hline waiting & 1000 & 50 & 15 & 2871 & 23253 \\
\hline connect & 1000 & 200 & 0 & 292 & 9206 \\
\hline processing & 1000 & 200 & 6255 & 53555 & 87348 \\
\hline waiting & 1000 & 200 & 6254 & 53455 & 87347 \\
\hline connect & 1000 & 400 & 0 & 222 & 9233 \\
\hline processing & 1000 & 400 & 188 & 71695 & 118069 \\
\hline waiting & 1000 & 400 & 156 & 42884 & 96568 \\
\hline
\end{tabular}

De acuerdo a los resultados obtenidos en las pruebas de carga para el servidor web podemos encontrar que es posible que accedan hasta 400 usuarios concurrentemente, y las pruebas se realizaron con 1000 peticiones. Al hacer las pruebas con más usuarios concurrentemente el sistema a veces colapsa.

\subsection{Evaluación mediante cuestionario de usabilidad}

El cuestionario de usabilidad se aplicó en dos Instituciones Educativas del nivel Primaria del área rural: la primera fue en la Institución Educativa "54461-Virgen del Carmen", con código modular "0200758", ubicada en la localidad de Saywite, en el distrito de Curahuasi de la Provincia de Abancay y Departamento de Apurímac, Perú; la segunda fue en la Institución Educativa "50040-Asillo", con código modular "0237354", ubicada en la localidad de Asillo, en el distrito y provincia de Abancay y Departamento de Apurímac, Perú. Los participantes fueron alumnos y docentes de cuarto, quinto y sexto grado del nivel primario. La aplicación del instrumento se llevó en el mes de abril del 2015. Participaron en total 38 personas, de los cuales 34 fueron estudiantes y 4 profesores. Los profesores contaban con varios años de experiencia en el aula.

El proceso de evaluación consistió en presentar y explicar el funcionamiento de la herramienta desarrollada al docente de aula. Luego el docente inició la sesión de aprendizaje correspondiente al tema de "fracciones" de la manera normal que lo realizaba antes, luego, utilizó las computadoras (Desktop y XO) del laboratorio para complementar la enseñanza, las cuales tenían acceso a la herramienta de animación por computadora denominada "math-fraccion".

Los docentes y alumnos utilizaron la herramienta aproximadamente por una hora, en la cual sin mucha explicación verbal, sino más bien cada uno de manera intuitiva empezó a explorar el recurso educativo. Empezaron a resolver los ejercicios planteados en cada uno de los niveles, y se sintieron familiarizados con las imágenes mostradas en la herramienta, porque eran fáciles de iden- 
tificar y estaban acorde con la realidad en que viven, ver Figura 8 y Figura 9.

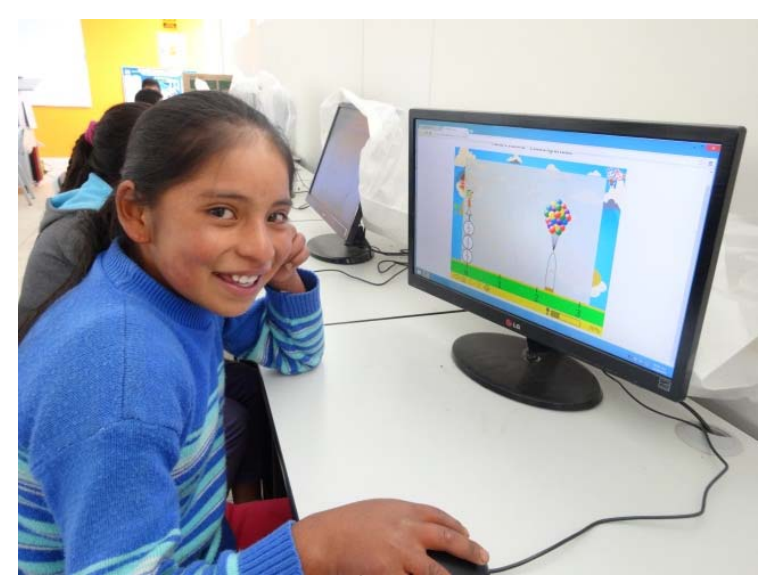

Fig. 8. Alumna utilizando una computadora en el laboratorio.

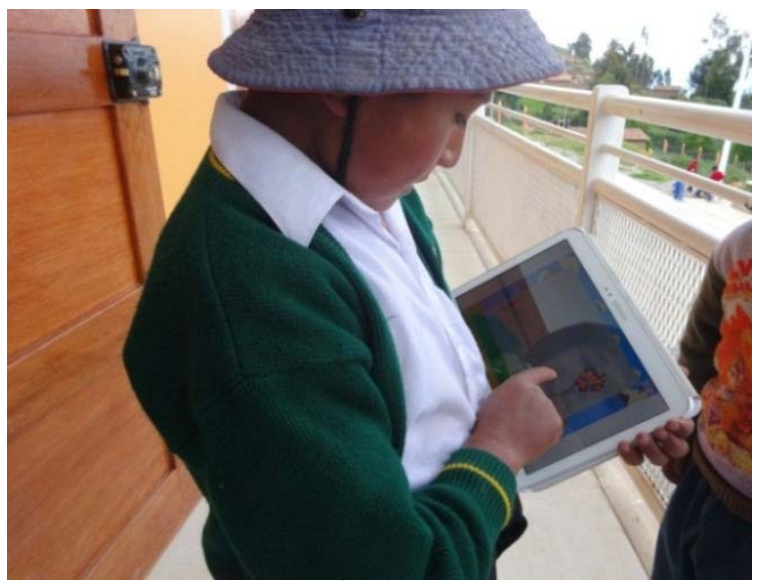

Fig. 9. Alumno utilizando una Tablet fuera del aula

Dentro de la Institución Educativa, los alumnos tuvieron acceso a la herramienta desarrollada mediante computadoras, laptop XO y dispositivos móviles. Luego completaron un cuestionario que buscaba evaluar la usabilidad y utilidad de la herramienta.

La Tabla 2 muestra los resultados del cuestionario aplicado a los docentes y alumnos que utilizaron la herramienta como parte del proceso experimntal. Como se mencionó antes, en dicha sesión de aprendizaje participaron 38 personas de la Institución Educativa Virgen del Carmen y Asillo. Los participantes respondieron utilizando una escala de likert de 1 a 10 , donde 1 representa "totalmente en desacuerdo" y 10 representa "totalmente de acuerdo".

La primera columna de la tabla muestra la variable "Nro. pers.", que representa el número de personas que ha dado una respuesta a una cierta pregunta. Luego se muestra la variable "min." que representa el valor mínimo obtenido; la variable "max." que representa el valor máximo asignado a ese ítem por parte de los participantes. La variable "Media" indica el promedio obtenido por la puntuación de cada participante. Finalmente la última columna de la Tabla 2 muestra la "Desv.Est." es la desviación estándar de los valores obtenidos.

TABLA 2

PREGUNTAS SOBRE LA USABILIDAD

\begin{tabular}{|c|c|c|c|c|c|}
\hline Pregunta & $\begin{array}{l}\text { Nro. } \\
\text { Pers. }\end{array}$ & $\begin{array}{l}\text { Valor } \\
\text { Min. }\end{array}$ & $\begin{array}{l}\text { Valor } \\
\text { Max. }\end{array}$ & Media & $\begin{array}{l}\text { Desv. } \\
\text { Est. }\end{array}$ \\
\hline 1)Me gusta el software & 38 & 7 & 10 & 8.37 & 1.24 \\
\hline $\begin{array}{l}\text { 2)Volvería a usar el soft- } \\
\text { ware }\end{array}$ & 38 & 8 & 10 & 9.08 & 0.85 \\
\hline $\begin{array}{l}\text { 3)Recomendaría el soft- } \\
\text { ware a otros amigos }\end{array}$ & 38 & 7 & 10 & 8.63 & 1.15 \\
\hline $\begin{array}{l}\text { 4)El software me muestra } \\
\text { información pertinente a } \\
\text { las matemáticas }\end{array}$ & 38 & 6 & 9 & 7.58 & 1.06 \\
\hline $\begin{array}{l}\text { 5)El software es fácil de } \\
\text { utilizar }\end{array}$ & 38 & 7 & 9 & 8.05 & 0.84 \\
\hline $\begin{array}{l}\text { 6)El software es diverti- } \\
\text { do }\end{array}$ & 38 & 7 & 10 & 8.71 & 1.23 \\
\hline $\begin{array}{l}\text { 7)El software es motiva- } \\
\text { dor }\end{array}$ & 38 & 7 & 9 & 8.00 & 0.81 \\
\hline $\begin{array}{l}\text { 8)El software se adapta a } \\
\text { mi ritmo }\end{array}$ & 38 & 3 & 8 & 5.39 & 1.69 \\
\hline $\begin{array}{l}\text { 9)El software me permi- } \\
\text { tió entender nuevas for- } \\
\text { mas de aprender mate- } \\
\text { máticas }\end{array}$ & 38 & 4 & 9 & 6.55 & 1.61 \\
\hline $\begin{array}{l}\text { 10)Me gustan los colores } \\
\text { que usa el diseño del } \\
\text { software }\end{array}$ & 38 & 6 & 10 & 8.00 & 1.45 \\
\hline $\begin{array}{l}\text { 11)Las imágenes del } \\
\text { software son claramente } \\
\text { identificables }\end{array}$ & 38 & 8 & 10 & 9.05 & 0.80 \\
\hline $\begin{array}{l}\text { 12)Las imágenes del } \\
\text { software me transmiten } \\
\text { información }\end{array}$ & 38 & 6 & 9 & 7.58 & 1.06 \\
\hline $\begin{array}{l}\text { 13)Los botones de des- } \\
\text { plazamiento son fáciles } \\
\text { de identificar }\end{array}$ & 38 & 3 & 9 & 5.87 & 2.12 \\
\hline $\begin{array}{l}\text { 14)Los botones de des- } \\
\text { plazamiento funcionan } \\
\text { correctamente. }\end{array}$ & 38 & 3 & 8 & 5.29 & 1.78 \\
\hline $\begin{array}{l}\text { 15)El texto mostrado en } \\
\text { la pantalla está bien di- } \\
\text { señado }\end{array}$ & 38 & 1 & 8 & 4.92 & 2.11 \\
\hline $\begin{array}{l}\text { 16)La distribución del } \\
\text { menú está bien diseñado }\end{array}$ & 38 & 3 & 8 & 5.29 & 1.78 \\
\hline $\begin{array}{l}\text { 17)El software muestra } \\
\text { información pertinente } \\
\text { para la enseñanza }\end{array}$ & 38 & 4 & 9 & 6.84 & 1.85 \\
\hline $\begin{array}{l}\text { 18)El software me podría } \\
\text { ayudar a enseñar o } \\
\text { aprender mejor }\end{array}$ & 38 & 7 & 9 & 8.03 & 0.75 \\
\hline
\end{tabular}

A continuación se analizan los resultados presentados en la Tabla 2:

Respecto al manejo de información visual. En los ítems 10) 11) y 12) se obtuvo un promedio de $8.00,9.05$ y 7.58 respectivamente. Esto nos muestra que los docentes y alum- 
nos coinciden en calificar positivamente la representación gráfica de los íconos, imagen y animación mostrada en la aplicación, particularmente aquellos usados en el software para representar las imágenes de acuerdo al contexto.

Respecto al apoyo en la enseñanza y aprendizaje de la matemática. En los ítems 4), 9), 17) y 18) se obtuvo un promedio de 7.58, 6.55, 6.84 y 8.03 respectivamente. Los docentes y alumnos califican positivamente el hecho de que la aplicación les permite tener mayores recursos didácticos como apoyo al proceso de enseñanza aprendizaje

Respecto a la satisfacción del usuario. En los ítems 1), 2) y 3) se obtuvo un promedio de $8.37,9.08$ y 8.63 respectivamente. Esto nos muestra que los participantes coinciden en calificar positivamente el hecho de que están satisfechos con el software desarrollado y les gusta la herramienta para la enseñanza y aprendizaje de las matemáticas.

Respecto al aspecto lúdico del software. En los ítems 5), 6) y 7) se obtuvo un promedio de $8.05,8.71$ y 8.00 respectivamente. Esto nos muestra que los participantes ven a la herramienta desarrollada como si fuera un juego y esto les divierte y motiva para su uso.

Respecto a los botones de navegación y menú. En los ítems $8), 13), 14), 15)$ y 16) se obtuvo un promedio de 5.39, 5.87, $5.29,4.92$ y 5.29 respectivamente. Los resultados muestran que los participantes coinciden en mostrar su poca conformidad con los botones de navegación y la disposición del menú. Esto probablemente es porque los usuarios todavía no están familiarizados con el uso de la forma gráfica y que no es necesario tener un menú tradicional y con bastante texto de por medio.

\subsection{Evaluación mediante opinión de Usuarios Expertos}

La evaluación en base a la opinión de usuarios expertos apuntó a determinar la usabilidad de la aplicación; específicamente el diseño de la interfaz de Math-Fraccion y su utilidad en el proceso de enseñanza aprendizaje como parte de una estrategia complementaria al método tradicional. Para realizar esta tarea se solicitó el apoyo de 3 usuarios expertos en usabilidad, ellos son ex $\bullet$ profesores auxiliares del curso de Taller de Usabilidad de Interfaces de Software, que imparte el Departamento de Ciencias de la Computación de la Universidad de Chile. Estas personas trabajan e investigan en temas de usabilidad de software, incluyendo el diseño de interfaces para dispositivos móviles.

El proceso de validación se inició con una breve explicación del objetivo para el cual se diseñó la herramienta, y el usuario final para la cual está diseñada. Seguidamente se realizó la simulación de una sesión didáctica de matemática para el tema de fracciones. Luego "los usuarios expertos" utilizaron la herramienta por 30 minutos aproximadamente.

Finalmente, se les solicitó que llenen un cuestionario en el cual daban su opinión respecto al diseño y usabilidad de la aplicación. Esto permitió obtener algunas observaciones y comentarios menores por parte de los expertos, los cuales fueron considerados para realizar el ajuste de la actual interfaz del sistema. A continuación se presentan los resultados obtenidos en ambas instancias de evaluación.

La Tabla 3 muestra los resultados obtenidos del cuestionario heurístico aplicado a los usuarios expertos, este cuestionario está basado en las 10 reglas heurísticas de usabilidad de Jakob Nielsen. Los participantes respondieron el cuestionario utilizando una escala de 1 a 5 , donde 1 representa "totalmente en desacuerdo" y 5 representa "totalmente de acuerdo".

Fueron dos los ítems mejor valorados por los usuarios expertos. El ítem Control del usuario y libertad, donde el valor promedio obtenido es de 5, lo cual indica que el usuario tiene el control del programa y la libertad para poder manipularlo. Luego el ítem Estética y diseño minimalista, donde el valor promedio obtenido es de 4.33 , lo cual indica que la interfaz del software es buena y tiene pocos elementos para su funcionamiento.

Por otro lado, se ha obtenido un promedio de 3.33 en dos ítems. El primero de estos ítems es Prevención de errores, donde el valor obtenido quiere decir que la aplicación previene los errores que normalmente podría cometer un usuario. El segundo es Reconocer en lugar de recordar, indica que el usuario puede reconocer visualmente los objetos en lugar de tener que memorizar.

Se ha obtenido un promedio de 3.0 en tres ítems que indica un término medio o neutro. El primer ítem es Flexibilidad y eficiencia de uso, lo cual indica que los accesos a las opciones del menú son fáciles e intuitivos. El segundo ítem se refiere a la relación entre el sistema y el mundo real, es decir, la aplicación tiene un lenguaje claro, los conceptos utilizados son entendibles y las palabras son de significado correcto. El tercer ítem es Reconocimiento, diagnóstico y recuperación de errores, esto implica que no hay un tratamiento explícito de para el tratamiento de los errores. $Y$ finalmente fueron tres los ítems menos calificados.

El ítem Consistencia y estándares se obtuvo un promedio de 2.00, uno de los puntajes más bajos, lo cual indica que existe poca consistencia y uso de estándares, esto probablemente porque la herramienta propuesta no se basa en el uso estándares, sino en la en la intuición del usuario. Similarmente, con un promedio de 2.67 puntos, uno de los más bajos, los evaluadores calificaron al ítem Ayuda y documentación, esto debido a que no se muestra información de ayuda incorporada en la herramienta, porque se considera que el enfoque es visual e intuitivo, por lo que no se muestra una documentación ni ayuda. De la misma manera en el ítem Visibilidad del estado del sistema, se obtuvo un valor de 2.33, quiere decir que la aplicación no muestra claramente al usuario en qué parte del sistema se encuentra, y que los enlaces están claramente señalados.

TABLA 3

Opinión de usuario experto

\begin{tabular}{|l|r|r|l|l|}
\hline \multicolumn{1}{|c|}{ Pregunta } & Min. & Máx. & $\begin{array}{l}\text { Me- } \\
\text { dia }\end{array}$ & $\begin{array}{l}\text { Desv } \\
\text { Est. }\end{array}$ \\
\hline $\begin{array}{l}\text { I. Visibilidad del estado } \\
\text { del sistema. }\end{array}$ & 2 & 3 & 2.33 & 0.58 \\
\hline $\begin{array}{l}\text { II. Relación entre sistema y } \\
\text { mundo real. }\end{array}$ & 2 & 4 & 3.00 & 1.00 \\
\hline III. Control del usuario y & 5 & 5 & 5.00 & 0.00 \\
\hline
\end{tabular}




\begin{tabular}{|l|r|r|l|l|}
\hline \multicolumn{1}{|c|}{ Pregunta } & Mín. & Máx. & $\begin{array}{l}\text { Me- } \\
\text { dia }\end{array}$ & $\begin{array}{l}\text { Desv } \\
\text { Est. }\end{array}$ \\
\hline libertad. & & & & \\
\hline $\begin{array}{l}\text { IV. Consistencia y estánda- } \\
\text { res. }\end{array}$ & 2 & 2 & 2.00 & 0.00 \\
\hline V. Prevención de errores. & 3 & 4 & 3.33 & 0.58 \\
\hline $\begin{array}{l}\text { VI. Reconocer en lugar de } \\
\text { recordar. }\end{array}$ & 2 & 4 & 3.33 & 1.15 \\
\hline $\begin{array}{l}\text { VII. Flexibilidad y eficien- } \\
\text { cia de uso. }\end{array}$ & 3 & 3 & 3.00 & 0.00 \\
\hline $\begin{array}{l}\text { VIII. Estética y diseño } \\
\text { minimalista. }\end{array}$ & 4 & 5 & 4.33 & 0.58 \\
\hline $\begin{array}{l}\text { IX. Reconocimiento, diag- } \\
\text { nóstico y recuperación de } \\
\text { errores. }\end{array}$ & 3 & 3 & 3.00 & 0.00 \\
\hline $\begin{array}{l}\text { X. Ayuda y documenta- } \\
\text { ción. }\end{array}$ & 2 & 4 & 2.67 & 1.15 \\
\hline
\end{tabular}

\section{Conclusiones y Trabajo Futuro}

Este artículo presenta un enfoque para la enseñanza de la matemática, específicamente para el tema de fracciones, la herramienta didáctica denominada "Math-fraccion", utiliza el concepto de "enfoque visual" para enseñar y aprender las matemáticas de manera divertida y motivadora, de tal manera que, el alumno puede profundizar sus conocimientos y hacer que su aprendizaje sea significativo.

Después de realizar las pruebas de usabilidad, una de las primeras conclusiones es que los docentes y alumnos consideran que Math-Fraccion les permite enseñar y aprender las matemáticas de manera divertida y de una manera distinta a las otras metodologías que ellos conocían. La enseñanza no es sólo verbal ni textual, sino es en forma visual y respetando los patrones culturales y sociales en el que se encuentran los usuarios. La mezcla de las animaciones y el juego hace que los alumnos muestren más interés en el aprendizaje y sienten que son parte del juego y definitivamente tienen una experiencia más enriquecedora.

Según los usuarios expertos en usabilidad, coinciden en manifestar que la herramienta didáctica "MathFraccion" tiene un buen diseño y estética minimalista, lo cual permite que el usuario acceda de manera rápida e intuitiva a cada una de las opciones del juego. Además, la herramienta es eficiente en el uso de recursos, porque realiza las tareas solicitadas en tiempos muy razonables, incluso en dispositivos con hardware limitado, como son las tablet y los teléfonos inteligentes.

Como parte del trabajo a futuro es necesario agregar a la herramienta más temas relacionados con las matemáticas, como por ejemplo: Sistema de numeración, operaciones básicas con números, etc. estos temas deberán estar de acuerdo al Diseño Curricular Nacional.

Finalmente, queda para los próximos estudios realizar pruebas de usabilidad y rendimiento más exigentes, y de la forma de visualizar la información sobre pantallas pequeñas en plataformas de Windows Mobile y Mac iOS. También queda pendiente probar la herramienta en nue- vos escenarios, como por ejemplo en Instituciones Educativas del norte y de la amazonia peruana.

\section{REFERENCES}

[1] PISA Reporte técnico Programme for International Student Assessment. Disponible en

http:/ / www.oecd.org/pisa/pisaproducts/PISA-2012-

technical-report-final.pdf. Último acceso 20 de abril del 2017.

[2] LLECE Reporte Técnico del Laboratorio Latinoamericano de Evaluación de la Calidad de la Educación. Disponible en: http:/ / portal.unesco.org/geography/es/ev.php-

URL_ID=13638\&URL_DO=DO_TOPIC\&URL_SECTION=201.h tml. Último acceso 20 de abril del 2014.

[3] ECE Reporte Técnico Evaluación Censal de Estudiantes. Disponible en: http:/ / noticia.educacionenred.pe/2017/04/resultados-ece2016-evaluacion-censal-estudiantes-umc-minedu-inei-gob-pe119627.html. Último acceso 20 de abril del 2017.

[4] A. B. Vera, "Las tecnologías de información y comunicaciones (TIC) en la docencia universitaria", Theoria, vol 12, no. 1, 109118, 2003.

[5] P. Jaramillo, P. Castañeda, and M. Pimienta, "Qué hacer con la tecnología en el aula: inventario de usos de las TIC para aprender y enseñar." Educación y Educadores, vol 12, no. 2, 2009.

[6] R. Ferreiro, "El reto de la educación del siglo XXI: la generación N." Apertura impresa no. 5, 2010.

[7] S. Castillo "Propuesta pedagógica basada en el constructivismo para el uso óptimo de las TIC en la enseñanza y el aprendizaje de la matemática". Revista latinoamericana de investigación en matemática educativa, vol. 11, no. 2, pp. 171-194, 2008.

[8] L. Torres, "Didáctica de las nuevas tecnologías de la información y la comunicación", Congreso Internacional Pedagogía, 2001.

[9] M. Reyes. "Los ordenadores en el proceso de enseñanzaaprendizaje de las ciencias: fundamentos para su utilización", Revista Iberoamericana de educación vol. 36, no.1, pp. 4, 2005.

[10] A. Sfard, "On the Dual Nature of Mathematical Conceptions: Reflections on Processes and Objects as Different Sides of the Same Coin," vol. 22, no. 1, pp. 1-36, 1991.

[11] A. Sierpinska, "Understanding in mathematics", Vol. 2, Psychology Press, 1994.

[12] R.B. Davis, "Learning mathematics: the cognitive science approach to mathematics education", Greenwood Publishing Group, 1984.

[13] Y. R. García, “Una ingeniería didáctica aplicada sobre fracciones" Omnia, vol. 13, no. 2, pp. 120-157, 2010.

[14] L. Pazos, "Las fracciones son un problema", Revista que hacer educativo, didáctica y practicas docentes, no. 97, p. 6, 2009.

[15] V. Pruzzo, “Las Fracciones: ¿Problema De Aprendizaje O Problemas De La Enseñanza?" Revista Pilquen, pp. 1-14, 2012.

[16] T. Kieren, D. Nelson and G. Smith, "Graphical Algorithms in Partitioning Tasks". The Journal of Mathematical. Behavior, no. 4. pp. $25-36,1985$.

[17] T. Kieren, "Rational and Fractional Numbers as Mathematical and Personal Knowled- ge: Implications for Curriculum and Instruction", Analysis of Arithmetic for Mathematics Teaching, New Jersey: Lawrence Erlbaun Associates, Publishers, vol 6, (323-369), 1992

[18] O. Figueras, "Dificultades de aprendizaje en dos modelos de enseñanza de los racionales". Tesis Doctoral. México: Cin- 
vestav-Matemática Educativa, 1988.

[19] O. Figueras, "Juntando partes. Hacia un modelo cognitivo y de competencia en la resolución de problemas de reparto", Didáctica Investigaciones en Matemática Educativa, México, Iberoamérica, pp. 173-196, 1996.

[20] M. Valdemoros, "La construcción del lenguaje de las fracciones y de los conceptos involucrados en él", Tesis Doctoral. México: Cinvestav-Matemática Educativa, 1993.

[21] M. Valdemoros, "Recursos intuitivos que favorecen la adición de fracciones: Estudio de caso. Educación Matemática", Grupo Editorial Iberoamérica Mexico, vol. 9, no. 3, pp. 5-17, 1997.

[22] M. Valdemoros, "Las fracciones, sus referencias y los correspondientes significados de la unidad", Grupo Editorial Iberoamérica, vol. 13, no.1, pp 51-67, 2001.

[23] A. Pitkethly, and R. Hunting, "Review of recent research in the area of initial fraction concepts", Educational Studies in Mathematics, vol. 30 no. 1, pp. 5-38, 1996.

[24] J.W. Bruce, P.J. Giblin, P.J. Rippon, “Microcomputers and mathematics" Cambridge University Press, 1990.

[25] E. Dubinsky, D. Tall, "Advanced mathematical Thinking and computer", En David Tall (ed) Advanced Mathematic Thinking, Mathematics Education Library, Netherlands, Kluwer Academic Publishers, 1991.

[26] M.K. Heid, “Transformation of learning of algebra and calculus via computer tools", 7th. International Congress on Mathematical Education. Microconference on calculators and computers. Quebec, Canada, 1992.

[27] J. J. Kaput, "Technology and Mathematics Education. Handbook of Research on Mathematics Teaching and Learning", A protect on NCTM, 1992.

[28] R. Morris, "Computer Experiments in a course for Mathematics Teachers", Journal of Computers in Mathematics and Science Teaching. vol. 11, no 1, pp. 13-18, 1992.

[29] J. Chang, "Practical applications and considerations of Visual teaching," ITME 2011 - Proc. 2011 IEEE Int. Symp. IT Med. Educ., vol. 2, pp. 453-455, 2011.

[30] A. Freire, D. Evandro, and D. Rodolfo, "Possibilidades de Ensino e Aprendizagem Por Meio da Elaboração de Uma Animação Interativa no Software Modellus", Conferencias LACLO, vol. 3 no. 1, 2012.

[31] C. Giovane, D. Salles, C. Boscarioli, G. Felipe, J. José, J. Paulo, and Q. De Aguiar, "Jogos Educativos Computacionais Como Objeto de Aprendizagem para o Ensino de Crianças Surdas em Fase de Alfabetização", Conferencias LACLO vol. 5, no. 1 pp. 313-321, 2014.

[32] L. F. Mota and E. P. Pimentel, "Jogo Digital para Motivar a Aprendizagem de Operações Aritméticas na Educação Básica", Conferencias LACLO, pp 155-165, 2014.

[33] E. V. B. Aguiar, "As novas tecnologias e o ensinoaprendizagem," Vértices, vol. 10, pp. 63-72, 2008.

[34] P. Woolner, "A Comparison of a Visual-Spatial Approach and a Verbal Approach To Teaching Mathematics", Proc. 28th Conf. Int. Gr. Psychol. Math. Educ., vol. 4, pp. 449-456, 2004.

[35] B. Moses, "Visualization: A different approach to problem solving" School Science and Mathematics 82, no. 2 pp. 141-147, 1992.

[36] H. Medhat, "From Visualization to Computer Animation Approaches in Mathematics Learning : the Legacy throughout History of Human Endeavours for Better Understanding" vol. 17, no. 4, pp. 279-290, 2013.
[37] Y. Chuang, "Teaching in a multimedia computer environment: A study of the effects of learning style, gender, and math achievement", Interactive Multimedia Electronic Journal of Computer-Enhanced Learning vol. 1, no. 1, 1999.

[38] Z. Felix, F. Junior, R. Oliveira, E. Siqueira, and M. Silva, “Objeto de Aprendizagem para o ensino da Matemática" pp. 658-664, 2006.

[39] A. Valdez, K. Trujillo, and K. Wiburg, "Math Snacks: Using Animations and Games to Fill the Gaps in Mathematics", Journal of Curriculum and Teaching, vol. 2, no. 2, pp. 154-161, 2013.

[40] E. V. B. Aguiar, "As novas tecnologias e o ensino-aprendizagem. Vértices", vol 10, no 1, pp 63-72, 2008.

[41] M. K. Rahmat, "The impact of computer animation learning toward students academic performance", In URL: www. unescobkk.org/fileadmin/user_upload/apeid/Conference/12t hConference/paper/2C2. Pdf, 2007.

[42] S. Aloraini, "The impact of using multimedia on students' academic achievement in the College of Education at King Saud University", Journal of King Saud University-Languages and Translation, vol 24, no 2, pp 75-82, 2012.

[43] Moyer-Packenham, P., \& Suh, J, Learning mathematics with technology: The influence of virtual manipulatives on different achievement groups. Journal of Computers in Mathematics and Science Teaching, 31(1), 39, 2012.

Manuel J. Ibarra, Ingeniero Informático y Sistemas en la Universidad Nacional de San Antonio Abad del Cusco en el 2002, Magister en Ciencias mención Computación en la Universidad de Chile en el 2011; docente en la Universidad Nacional Micaela Bastidas de Apurímac; segundo mejor artículo en LACLO 2015 Brasil; 9 artículos indexados por scopus.

Yonatan Mamani. Ingeniero Informático y Sistemas en la la Universidad Nacional Micaela Bastidas de Apurímac; profesor contratado en la Universidad Nacional Micaela Bastidas de Apurímac.

Pablo E. Ataucusi. Licenciado en Educación especialidad Matemática e Informática en la la Universidad Nacional Micaela Bastidas de Apurímac; profesor en el Colegio de alto Rendimiento de Abancay; segundo mejor artículo en LACLO 2015 Brasil.

Carmen Palomino. Licenciada en Educación Universidad Nacional San Cristobal de Huamanga; Doctorado en Ciencias de la Educación Universidad Nacional Daniel Alcides Carrión de Cerro de Pasco; docente en la Universidad Tecnológica de los Andes.

Vladimiro Ibañez. Ingeniero Estadístico en la Universidad Nacional del Altiplano, Magister Scientiae en Informatica en la Universidad Nacional del Altiplano, docente en la Universidad Nacional del Altiplano. 